\title{
Ciberadicción y rendimiento académico en adolescentes del distrito de Mariscal Benavides, Amazonas, Perú. 2021
}

\section{Cyber addiction and academic performance in adolescents from the Mariscal Benavides district, Amazonas, Peru. 2021}

\author{
Emily Huamán Guiop ${ }^{1}$, Victor Edwin López Sánchez ${ }^{2}$, Jhasill Tafur Fernández ${ }^{3}$
}

\section{RESUMEN}

El objetivo de la investigación fue establecer el nivel de relación entre ciberadicción y rendimiento académico en adolescentes del distrito de Mariscal Benavides, provincia de Rodríguez de Mendoza, Amazonas, Perú, 2021, el enfoque fue cuantitativo, relacional. Se utilizó el muestreo no probabilístico por conveniencia, la muestra estuvo conformada por 21 adolescentes. Para la recolección de datos se utilizó como instrumento para la ciberadicción la "Escala de ciberadicción de Lima”, el cual presentó 11 ítems que evaluaron, características sintomatológicas y características funcionales. Para el rendimiento académico se utilizó la libreta de notas que contenía el promedio obtenido de los estudiantes por curso, del año 2020 y para establecer la relación entre las variables se utilizó el coeficiente de correlación de Pearson. Los resultados mostraron que el 14.3\% (3) de los adolescentes presenta un nivel bajo de ciberadicción y el 85.7\% (18) un nivel moderado, mientras que en rendimiento académico el 52.4\% (11) presenta un nivel regular y el 47.6\% (10) presenta nivel excelente. Concluyendo que existe una correlación negativa moderada $\mathrm{r}=-0.366$ entre ciberadicción y rendimiento académico.

Palabras clave: Adicción, internet, adolescentes, rendimiento académico

\begin{abstract}
The objective of the research was to establish the level of relationship between cyber addiction and academic performance in adolescents from the Mariscal Benavides district, Rodríguez de Mendoza province, Amazonas, Peru, 2021, the approach was quantitative, relational. Non-probability sampling was done for convenience, the sample consisted of 21 adolescents. For data collection, the "Lima Cyberdiction Scale" was used as an instrument for cyber addiction, which presented 11 items that evaluated symptomatological characteristics and functional functions. For academic performance, the grade book containing the average obtained from the students per course of the year 2020 was used and to establish the relationship between the variables, the Pearson correlation coefficient was used. The results showed that 14.3\% (3) of adolescents present a low level of cyber addiction and $85.7 \%$ (18) a moderate level, while in academic performance 52.4\% (11) present a regular level and $47.6 \%$ (10) presents an excellent level. Concluding that there is a moderate negative correlation $r=-0.366$ between cyber addiction and academic performance.
\end{abstract}

Keywords: Addiction, Internet, Adolescents, Academic performance

\footnotetext{
${ }^{1}$ Estudiante de pregrado de la carrera profesional de psicología. Facultad de Ciencia de la Salud de la Universidad Nacional Toribio Rodríguez de Mendoza. Correo electrónico: emilyhuaman8@gmail.com

${ }^{2}$ Estudiante de pregrado de la carrera profesional de psicología. Facultad de Ciencia de la Salud de la Universidad Nacional Toribio Rodríguez de Mendoza. Correo electrónico: lopezsanchezvictoredwin@gmail.com

${ }^{3}$ Estudiante de pregrado de la carrera profesional de psicología. Facultad de Ciencia de la Salud de la Universidad Nacional Toribio Rodríguez de Mendoza. Correo electrónico: jhasill.tafur@gmail.com
} 


\section{INTRODUCCIÓN}

Desde la aparición de internet remontado aproximadamente al año de 1983, este ha ido adquiriendo grandes cambios, convirtiéndose en el eje de la vida cotidiana pues existen múltiples estudios que reafirman el uso masivo de internet principalmente por la población adolescente. El inicio de la manipulación de aparatos electrónicos empieza desde una edad muy temprana, con el fin de entretener a los niños mientras los padres o cuidadores realizan labores del hogar, de este modo si bien el internet puede facilitarnos la vida, puesto que simplifica aquellas tareas que antes nos parecían complejas; también puede acarrear consecuencias negativas si se llega a abusar de su uso.

Se han realizado investigaciones acerca de este tema, pero resulta difícil establecer una definición exacta de lo que es ciberadicción ya que es conocido con diferentes términos (tecnoadicciones, uso compulsivo o patológico de internet), para ello Young define de manera más precisa lo que vendría a ser la adicción a internet considerándolo como un deterioro presentado por ciertas señales manifestadas en la esfera psicológica, conductual y fisiológica del individuo ya que aquellos que presentan un uso excesivo de internet presentan problemas dentro de su vida personal, familiar, social, académico (citado por Terán, 2019).

Por otro lado, en cuanto al rendimiento académico, este se conoce como una problemática que ha venido existiendo desde tiempos remontables generando preocupación en nuestra sociedad; Caballero y sus colaboradores mencionan que el rendimiento académico involucra la consecución de los objetivos señalados dentro de la institución educativa o el curso asignado a los estudiantes y esto se puede reflejar mediante las notas que cada uno obtiene (Citado por Lamas, 2015).

El problema de ciberadicción y su afección en la esfera biopsicosocial del individuo no es un inconveniente que solo sucede en Perú, sino también alrededor del mundo (Sharma, et al 2021) donde se da a conocer que aquellos que se encuentran conectados por mucho tiempo, presentan síntomas muy similares al síndrome de abstinencia, afectando a los adolescentes en diversas áreas de su vida, (Rojas, et al. 2018), teniendo dicho acceso a través de distintos medios como celulares, computadoras, tabletas interviniendo en el actuar diario de los seres humanos (Cobacango, et al. 2019), considerándose también como un problema educativo actual puesto que interfiere en el buen funcionamiento del proceso enseñanza-aprendizaje (Sánchez y Zambrano, 2020).

En la actualidad los adolescentes se ven inmersos en el mundo tecnológico llegando a existir una relación estadística significativa entre ciberadicción y rendimiento académico, puesto que muchos niños, adolescentes, adultos descuidan sus metas para seguir en este mundo cibernético, por diversas razones que les genera satisfacción instantánea y aún la sociedad no es consciente del daño a futuro

\section{MATERIAL Y MÉTODO}

Esta investigación fue de diseño transversal, y orientación básica, con un enfoque cuantitativo, de alcance temporal sincrónico. De método de investigación no experimental y de tipo correlacional.

Se utilizó el muestreo no probabilístico por conveniencia donde participaron 21 adolescentes estudiantes del distrito Mariscal Benavides, considerando lo siguiente:

Los criterios de inclusión fueron: Estudiantes de secundaria, adolescentes cuya edad comprende desde los 14 a 18 años de edad, estudiantes que reenviaron de forma afirmativa el consentimiento informado, excluyendo a los adolescentes que no disponían de celular, computadora, tablet, acceso a internet o adolescentes que sean padres de familia.

Para medir el nivel de ciberadicción en los adolescentes del distrito de Mariscal Benavides se utilizó a La Escala de Adicción al Internet de Lima (EAIL); esta prueba se creó como un contraste de medir la manifestación de la ciberadicción en adolescente, con 11 ítems los cuales evaluaron dos dimensiones: Características sintomatológicas y Características funcionales; cada ítem presentó alternativas de respuestas, con escala de tipo Likert, con la siguiente valoración: Muy rara vez $=1$ punto, Rara vez $=2$ puntos, A menudo $=3$ puntos y Siempre $=4$ puntos. La puntuación de esta escala varía entre 0 y 44 puntos. La EAIL presentó un coeficiente de confiabilidad del alfa de Cronbach de 0,84 .

Para medir el rendimiento académico se utilizó formularios de Google forms que permitieron conocer las notas de 10 cursos académicos validos en el currículo de estudio peruano, utilizando la sumatoria de las mismas se procedió a colocar en una escala de medición los puntajes obtenidos. El rango de 10-26 fue de clasificación bajo, de 27-42 regular y de 43-60 excelente.

La aplicación de los instrumentos se dio de manera individual y virtual (debido a la situación actual de COVID-19), enviados a través de cuestionarios de Google Forms a los correos personales de cada estudiante, para utilizar la información proporcionada por los adolescentes en el estudio, se requirió el consentimiento informado de cada uno de los participantes, dicho documento fue enviado mediante la plataforma de Google Forms para su aceptación. 
Para comprobar la relación entre variables de estudio se aplicó el coeficiente de correlación de Pearson.

\section{RESULTADOS}

Tabla 1

Correlación entre las variables ciberadicción y rendimiento académico en adolescentes del del distrito de Mariscal Benavides, Amazonas, Perú. 2021.

\begin{tabular}{c|c|c|c}
\hline Variables & & CA & RA \\
\hline $\begin{array}{c}\text { Ciberadicción } \\
\text { (CA) }\end{array}$ & $\begin{array}{c}\text { Correlación } \\
\text { (bilateral) }\end{array}$ & - & -0.366 \\
& Significancia & - & .103 \\
\cline { 2 - 4 } $\begin{array}{c}\text { Rendimiento } \\
\text { Académico } \\
\text { (RA) }\end{array}$ & $\begin{array}{c}\text { Correlación } \\
\text { (bilateral) }\end{array}$ & -0.366 & - \\
Significancia & .103 & - \\
\hline Total & \multicolumn{4}{c}{ N } \\
\hline$* *$ La correlación esegativa moderada.
\end{tabular}

En la tabla 1 se observa el nivel de significancia del coeficiente de correlación de Pearson, con un valor mayor a $0.05(p=0.103>0.05)$, lo que significa, que no hay correlación estadísticamente significativa entre las variables ciberadicción y rendimiento académico, es decir, se acepta la hipótesis nula. Así mismo, el valor del coeficiente de correlación, presentó un signo negativo $(\mathrm{r}=-0.366)$, lo que quiere decir, que existe una dirección inversamente proporcional entre las dos variables, donde la fuerza de asociación es moderada negativa; lo que significa, que a mayor ciberadicción menor será el rendimiento académico y que, a menor ciberadicción mayor será el rendimiento académico.

\section{Tabla 2}

Nivel de Ciberadicción en los adolescentes del distrito de Mariscal Benavides, Amazonas, Perú, 2021 .

\begin{tabular}{ccc}
\hline Nivel de Ciberadicción & fi & \% \\
\hline Bajo & 3 & 14.3 \\
Moderado & 18 & 85.7 \\
Alto & 0 & 0.0 \\
\hline Total & $\mathbf{2 1}$ & $\mathbf{1 0 0 \%}$ \\
\hline
\end{tabular}

En la tabla 2 se observa que, del total de 21 adolescentes encuestados en el distrito de Mariscal Benavides 3(14,3\%) presentan un nivel bajo de Ciberadicción, seguido de $18(85,7 \%)$ adolescentes que presentan un nivel moderado de Ciberadicción y $0 \%$ no evidenciaron niveles altos de ciberadicción.

\section{Tabla 3}

Nivel de Rendimiento Académico en adolescentes del distrito de Mariscal Benavides, Amazonas, Perú. 2021.

\begin{tabular}{ccc}
\hline $\begin{array}{c}\text { Nivel de Rendimiento } \\
\text { Académico }\end{array}$ & fi & $\%$ \\
\hline Excelente & 10 & 47.6 \\
Regular & 11 & 52.4 \\
Bajo & 0 & 0.0 \\
\hline Total & $\mathbf{2 1}$ & $\mathbf{1 0 0 \%}$ \\
\hline
\end{tabular}

En la tabla 3 se muestra que, de los 21 adolescentes del distrito de Mariscal Benavides, que participaron en el estudio, $11(52.4 \%)$ estudiantes evidencian un nivel regular correspondiente al rendimiento académico, mientras que, $10(47.6 \%)$ acreditan estar a un nivel de excelencia, y ningún estudiante ( $0 \%$ ) se encuentra en un nivel bajo, respecto al rendimiento académico.

\section{DISCUSIÓN}

En la investigación se tuvo la participación de 21 adolescentes del distrito de Mariscal Benavides, provincia de Rodríguez de Mendoza, Amazonas donde el 14.3\% (3) de los encuestados presentan un nivel bajo de Ciberadicción, es decir los adolescentes tienen un control en cuanto al uso de internet por parte de sus padres o no cuentan con un celular propio con el cual navegar de forma ilimitada. Asimismo, el $85.7 \%$ (18) de los adolescentes reflejaron un nivel moderado de Ciberadicción dando a conocer que cuentan con seguimiento y supervisión de sus padres para el uso adecuado del internet y $0 \%$ no evidenciaron niveles altos de ciberadicción, es decir no existen adolescentes dentro de la muestra tomada en cuenta que sean adictos a su uso.

Zevallos (2020). Perú. En su estudio realizado en Lima a 335 adolescentes de una Institución educativa pública, para determinar el nivel de ciberadicción, manifestó que $151(45 \%)$ de adolescentes se encuentran en un nivel alto, $127(38 \%)$ en un nivel muy alto y 57 (17\%) del total de 335 adolescentes se encuentran en un nivel medio de Ciberadicción, y finalmente $0 \%$ de adolescentes se encuentran en un nivel bajo.

Por otro lado, López, Vega, et al. (2019). Colombia. En su investigación enfocado a estudiantes de nivel secundario entre 13 a 15 años, obtuvieron como resultado que 69 adolescentes presentan ciberadicción a un nivel normal, 22 moderado y 56 adolescentes se encuentran en un nivel bajo.

En cuanto a la variable ciberadicción, el resultado dominante se reflejó en el nivel moderado. Dicho 
resultado no coincide con lo encontrado por Zevallos (2020) puesto que en su investigación predomina el nivel alto con la mayor cantidad de los adolescentes. Sin embargo, los resultados obtenidos se asemejan a lo encontrado por López, Vega, et al. (2019) donde predominan el nivel moderado y normal. Los resultados obtenidos en las investigaciones tomadas en cuenta pueden variar debido a distintos factores, como el lugar de procedencia, la velocidad de acceso a internet, etc.

En cuanto al rendimiento académico, en el presente estudio se obtuvo que el $52.4 \%$ (11) estudiantes evidencian un nivel regular, lo que indica que los adolescentes no presentan un buen desempeño académico en las distintas áreas dictadas dentro de su casa de estudios; mientras que el 47.6\% (10) acreditan estar a un nivel de excelencia, demostrando buenas calificaciones en los 11 cursos tomados en cuenta y en lo que concierne al nivel bajo no se evidenciaron estudiantes dentro de este rango.

Ramos (2020). Perú. En su estudio para determinar el nivel de rendimiento académico en 149 adolescentes de una Institución educativa secundaria en Puno, obtuvo como resultado que $80(53.7 \%)$ se encontraban en un nivel bueno, $49(32.9 \%)$ en un nivel regular y 20 (13.4\%) en un nivel de rendimiento académico malo.

Carrazco, Martínez, et al (2019). Perú. En su investigación para conocer el nivel de Rendimiento académico en 234 adolescentes de 13 a 16 años en Huánuco, obtuvieron como resultado que $12(5.1 \%)$ adolescentes presentaron un nivel deficiente de rendimiento académico, $53(22.6 \%)$ un nivel bajo, $129(55.1 \%)$ un nivel medio y $40(17.1 \%)$ presentaron un nivel alto en cuanto al rendimiento académico.

Castro (2019). Perú. En su investigación realizado en Lima para conocer el nivel de rendimiento académico, donde la muestra total fue 90 adolescentes, obtuvo como resultado que 10 (11\%) adolescentes presentan un nivel alto, 79 (88\%) nivel medio y $1(1 \%)$ adolescente que se encuentra en un nivel bajo en cuanto al rendimiento académico.

Los resultados obtenidos en la variable rendimiento académico, se encuentra en discrepancia con el estudio realizado por Ramos (2020), puesto que en su investigación predomina el nivel bueno con más de la mitad de los estudiantes dentro de esta categoría, a su vez se evidenció un porcentaje considerable dentro de un nivel malo en comparación con el estudio realizado. Sin embargo, lo presentado por Carrazco, Martínez, et al (2019) y Castro (2019) coinciden con lo obtenido en la investigación, donde impera el nivel medio con la mayor parte de los estudiantes ubicados en esta categoría.

En cuanto a los resultados encontrados dentro de esta investigación queda demostrado que la ciberadicción y el rendimiento académico tienen una correlación negativa moderada cuyo valor es $r=-0.366$, es decir que, a mayor ciberadicción, menor será el rendimiento académico o que a menor ciberadicción, mayor será el rendimiento académico.

Mientras que Milla y Rojas (2018), Perú. Obtuvieron en su investigación una correlación de Rho Spearman $=-0,867$ donde concretaron que la relación entre ambas variables es muy fuerte teniendo una relación negativa.

Machaca (2017), Perú. También evidencia en su estudio una dependencia negativa moderada en el que muestran un $r=-0,767$, el cual revela que a mayor adicción a internet el nivel del juicio lector en el área de comunicación es muy deficiente; evidenciando también que el $13 \%$ de la muestra corresponde a estudiantes ciberadictos y el $72 \%$ se encuentra en riesgo.

Comparando con las investigaciones mencionadas el presente estudio, refleja coincidencia, puesto que existe una relación negativa moderada entre la variable ciberadicción y rendimiento académico.

Así mismo, dentro del desarrollo de la investigación se presentaron ciertas limitaciones, como el trabajar con una muestra reducida de adolescentes, debido a la poca disposición de los estudiantes y a la coyuntura actual COVID-19.

\section{CONCLUSIONES}

Mediante el coeficiente de correlación de Pearson $(\mathrm{p}>0.05)$ se encontró que existe una relación negativa moderada entre la variable ciberadicción y la variable rendimiento académico.

El nivel de mayor predominio en cuanto a la variable ciberadicción es el nivel moderado con el 85.7\% (18) de los adolescentes.

El nivel de mayor predominio respecto a la variable rendimiento académico, es el nivel medio con el $52.4 \%$ (11) de los adolescentes.

\section{REFERENCIAS BIBLIOGRÁFICAS}

Carrazco, M., Cecilia, M., Noreña, F., \& Bao, C. (2020). Satisfacción familiar y rendimiento académico en adolescentes de un Colegio Estatal de Huánuco, Perú. Boletín Redipe, 9(2), 197-210. https://dialnet.unirioja.es/ servlet/articulo?codigo $=7528387$

Castro, M. (2020). Autoeficacia y rendimiento académico en adolescentes de un centro de educación técnico productiva, Lima. https://repositorio.ucv.edu.pe/bitstream/han dle/20.500.12692/41607/Castro_SM.pdf?s 
Cobacango, J., Cedeño, V., \& Tinoco, M. (2019). La ciberadicción en la conducta de los estudiantes. Revista Atlante: Cuadernos de educación y desarrollo. Obtenido de https://www.eumed.net/rev/atlante/2019/07/ ciberadiccion-estudiantes.html

Lamas, H. (2015). Sobre el rendimiento escolar. Propósitos y Representaciones, 3(1), 313386. doi:http:dx.doi.org/10.20511/pyr 2015.v3n1.74

López, S., Vega, H., \& Suárez, D. (2019). Ciberadicción en Adolescentes En Girón; Santander. https://repository.ucc.edu. co/bitstream/20.500.12494/15501/1/2019_c iberadiccion_ninos_adolescentes.pdf

Ramos, R. (2020). Rendimiento academico en estudiantes de la Institución educativa secundaria "Juan Bustamante Dueñas" Puno. http://repositorio.unap.edu.pe /bitstream/handle/UNAP/13626/Ramos Ro ger_Mamani_Clipsania.pdf?sequence $=1 \&$ is Allowed $=\mathrm{y}$

Rojas, C., Ramos, J., Pardo, E., \& Henríquez, F. (2018). Adicción a internet en adolescentes: una breve descripción. Drugs and Addictive Behavior , 3(2), 267-281. doi: http://dx. doi.org/10.21501/24631779.2876

Sánchez, B., \& Zambrano, G. (2020). La ciberadicción en el rendimiento académico de los estudiantes de educación básica superior de la escuela Cicerón Robles Velásquez, 2019. Revista Caribeña de Ciencias Sociales. https://www.eumed.net/ rev/caribe/2020/08/ciberadiccion.html

Sharma, P., Kumar, A., Lamba, R., \& Awasthi, A. (2021). Internet addiction and academic performance among high school urban students. International Journal of Health and Clinical Research, 4(6), 237-240. https://ijhcr.com/index.php/ijhcr/article/vie w/1256/1057

Terán, P. (2019). Ciberadicciones, adicción a las nuevas tecnologías (NTIC). Asociación Española de Pediatría de Atención Primaria, 131-142. https://www.aepap.org/sites /default/files/pags._131-142_ciberadi cciones

Zevallos, I. (2020). Ciberadicción en adolescentes de una Institución educativa pública de Ate. https://repositorio.ucv.edu.pe/bitstream/han dle/20.500.12692/54976/Zevallos_RIJ\%20- 